\title{
LOW-RATE POISSON INTENSITY ESTIMATION USING MULTIPLEXED IMAGING
}

\author{
Dongeek Shin Ahmed Kirmani Vivek K Goyal \\ Research Laboratory of Electronics \\ Massachusetts Institute of Technology
}

\begin{abstract}
Multiplexed imaging is a powerful mechanism for achieving high signal-to-noise ratio (SNR) in the presence of signal-independent additive noise. However, for imaging in presence of only signaldependent shot noise, multiplexing has been shown to significantly degrade SNR. Hence, multiplexing to increase SNR in presence of Poisson noise is normally thought to be infeasible. In this paper, we present an exception to this view by demonstrating multiplexing advantage when the scene parameters are non-negative valued and are observed through a low-rate Poisson channel.
\end{abstract}

Index Terms-Multiplexed illumination and sensing, image denoising, shot noise, photon-limited imaging, Poisson noise, signaldependent noise, convex optimization.

\section{INTRODUCTION}

Multiplexed illumination or sensing is frequently used in imaging applications to obtain significant improvement in signal-to-noise ratio (SNR) in the presence of signal-independent additive noise. For example, in [1] an approach based on Hadamard codes was introduced to image objects under variable-direction lighting. The setup for this problem is shown in Figure 1.

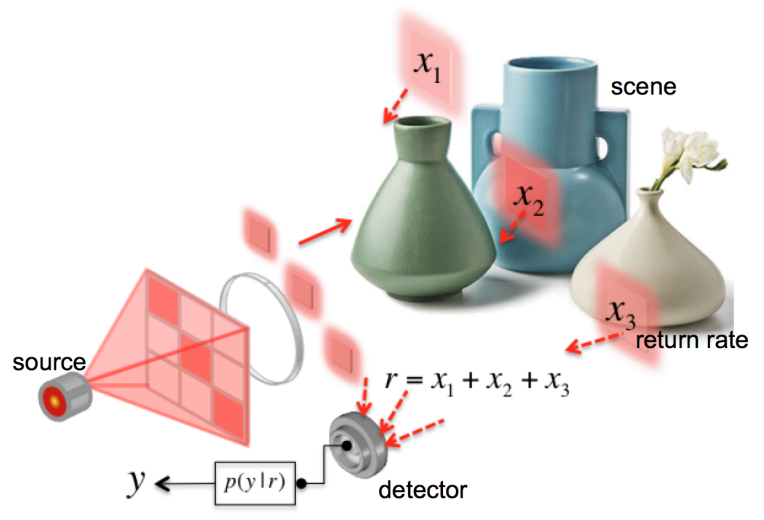

Fig. 1. Multiplexed imaging setup

Multiplexed imaging comprises two stages: In the first stage, a sequence of multiplexed images is acquired by illuminating the scene simultaneously using different subsets of multiple sources. For each image in the acquisition sequence, the pattern of on sources is based on a code matrix. In the second stage, to recover the images that

This material is based upon work supported by the National Science Foundation under grant No. 1161413, a Qualcomm Innovation Fellowship, and a Samsung Scholarship. correspond to only one on source at a time, the acquired image sequence is demultiplexed, ordinarily using simple code matrix inversion. Having more than one on source at a time increases object irradiance, and assuming only signal-independent additive noise in the observations, the demultiplexed images obtained by code matrix inversion have enhanced SNR relative to images that are acquired traditionally by turning on one light source at a time. Multiplexed imaging also reduces the effect of additive background or sensor dark current [1]. Note that multiplexing does not decrease the number of measurements required. Multiplexed imaging still requires as many measurements as the number of illumination sources. Also, the demultiplexed images are produced without the use of scene priors or spatial regularization.

It is a well known fact that, multiplexed imaging solely in the presence of Poisson noise, where demultiplexing is done using matrix inversion, substantially degrades the SNR [2] (see Section 3.2). Related work on multiplexed imaging in the presence of signal-dependent noise [3] assumes two noise components in the observation channel: Poisson noise and additive white Gaussian noise (AWGN). The approach in [3] focuses on using multiplexed illumination to mitigate the effect of AWGN. In this case, demultiplexing amplifies the effect of signal-dependent Poisson noise but reduces the effect of signal-independent AWGN. The technique in [3] obtains an overall increase in SNR when AWGN is the dominant source of noise; but the method fails when Poisson noise is the dominant noise source.

In this paper, we present the following results for multiplexed imaging in the case when Poisson or shot noise is the only source of noise:

1. We prove that for non-negative signals like image intensities, demultiplexing using matrix inversion is not equal to the maximum likelihood intensity estimate (MLIE).

2. Using simulations, we show that when image intensity observations are made using low rate Poisson channels, MLIE using multiplexed measurements has significant SNR improvement over the traditional acquisition technique of measuring one channel at a time.

The enhanced SNR in Claim 2 does not contradict the well-known negative result about SNR degradation due to multiplexing in presence of Poisson noise [2]; this is because the degradation of SNR is in context of demultiplexing using code matrix inversion and not with maximum likelihood estimation. As we will show in Section 4, the two approaches-matrix inversion and ML-are equivalent when either there are no non-negativity constraints on the intensity signal, or the intensity measurements are made through a high-rate Poisson channel. The authors are unaware of previously published work that compares the two methods of demultiplexing.

The low-rate Poisson intensity estimation problem is applicable in a number of practical scenarios such as astronomy, night vision, 

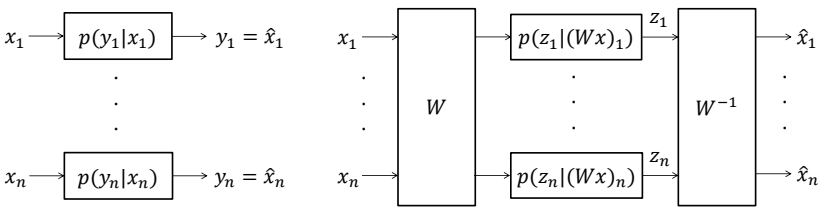

Fig. 2. Block diagram of setups measuring direct illumination (left) and multiplexed illumination (right) over independent noisy channels.

medical imaging applications such as positron emission tomography, and imaging of light sensitive biological and chemical samples. In these applications, the measurements are often collected using single photon-counting detectors. The observations obtained from such detectors are inherently noisy due to low count levels, and corrupted by signal-dependent Poisson noise [4].

Note that our interest here is in classical estimation rather than Bayesian estimation. For cases where a non-trivial prior is available, the maximum a posteriori probability estimator would presumably have some of the same behavior as we find in this paper for the ML estimator. We also do not differentiate between computational methods for finding the ML estimate; we use SPIRAL-TAP [5] without the option of spatial regularization to solve the non-negatively constrained MLIE problem, though generalized approximate message passing [6] also facilitates inclusion of Poisson likelihoods.

\section{PROBLEM FORMULATION}

Let $x$ be a size- $n$ vector that represents the scene intensities. Consider the setup where each of the $n$ light sources illuminates a scene point and the reflected light is collected by a single bucket detector.

The traditional illumination method involves turning each light source on, one at a time and acquiring $n$ measurements in total. In contrast, the multiplexed illumination method acquires a sequence of $n$ measurements obtained by turning on multiple light sources at the same time using coded illumination patterns.

All the measurements are observed over independent noisy channels. We consider two kinds of noise models: signal-independent additive Gaussian and signal-dependent Poisson. The goal is to estimate the original signal, $x$, based on the measurements.

As stated, in the traditional illumination method each of the $n$ sources are turned on one-by-one resulting in observations $y_{i}=x_{i}+$ $\eta_{i}$ for $i \in\{1,2, \ldots, n\}$. Let $\eta_{i}$ be additive zero-mean Gaussian noise with standard deviation $\sigma$. Let $W$ denote the code matrix used for multiplexed illumination and let $w_{i}^{T}$ denote its rows (see Figure 2).

$W$ is typically defined as a $(0,1)$-matrix with every row describing a certain pattern of turning sensors on and off. Multiplexed illumination leads to the observation $z_{i}=w_{i}^{T} x+\eta_{i}$ for $i \in\{1,2, \ldots, n\}$. When the noise model is Poisson, we observe $y_{i}=\operatorname{Poi}\left(x_{i}\right)$ under traditional illumination and $z_{i}=\operatorname{Poi}\left(w_{i}^{T} x\right)$ under multiplexed illumination.

In acquisition using traditional illumination, the signal, $x$, is estimated based on maximizing the likelihood function $p(y \mid x)$. The maximum likelihood estimate of $x$ is then $\hat{x}=y$. With multiplexing, the estimate of illumination is based on $p(z \mid W x)$ and is equal to the matrix inverse solution $\hat{x}=W^{-1} z$ when the signal $x$ is not subject to any constraint, such as non-negativity.

\section{PRIOR WORK}

\subsection{Multiplexed imaging under signal-independent additive Gaussian noise}

Let $\hat{x}$ be an estimate of the original signal $x$. The purpose of multiplexed sensing is to reduce the mean square error (MSE) in estima- tion. We define $\operatorname{MSE}(\hat{x})=\operatorname{tr}\left[\mathbb{E}(x-\hat{x})(x-\hat{x})^{T}\right]$. The multiplexing gain associated with using a multiplexing code $W$ is defined as the reduction ratio in the root MSE:

$$
G(W)=\sqrt{\frac{\operatorname{MSE}\left(\hat{x}_{\mathrm{direct}}=y\right)}{\operatorname{MSE}\left(\hat{x}=W^{-1} z\right)}}
$$

$G>1 \Rightarrow$ multiplexed imaging results in better signal estimation. Previous work [1] assumes AWGN observation channels. Let $\eta$ be the additive Gaussian noise vector and $W$ be a multiplexing matrix. Then for measurements acquired in presence of AWGN we have,

$$
G(W)=\sqrt{\frac{\operatorname{tr}\left[\mathbb{E}\left(\eta \eta^{T}\right)\right]}{\operatorname{tr}\left[\mathbb{E}\left(W^{-1} \eta \eta^{T} W^{-T}\right)\right]}}=\sqrt{\frac{1}{\operatorname{tr}\left[\left(W^{T} W\right)^{-1}\right]}} .
$$

Designing multiplexing matrices based on Hadamard codes are optimal in the sense that, for fixed $n$, $\arg \max _{W}(G(W))=H$ under AWGN [7]. A Hadamard multiplexing matrix $H$ of size $n \times n$ is constructed by deleting the first row and column of a Hadamard matrix of size $n+1$ and replacing 1's with 0's and -1 's with 1's [8]. It can be shown that $G(H)=(n+1) /(2 \sqrt{n})$. Since $n>1$ implies $G(H)>1$, multiplexed imaging using Hadamard codes achieves the best signal reconstruction over any other multiplexing code, including traditional acquisition.

\subsection{Multiplexed imaging under Poisson noise}

We are interested in photon-limited imaging, where the noise corruption is dominated by Poisson noise. Measurements for traditional imaging are denoted by $y_{i} \sim \operatorname{Poi}\left(x_{i}\right)$ for $i \in\{1,2, \ldots, n\}$. A strictly positive $x$ can be safely assumed in practice as background intensity cannot be perfectly eliminated.

We now show that Hadamard multiplexing followed by matrix inversion severely degrades the signal-to-noise ratio and increases the MSE in signal estimation. This is because Poisson noise from multiplexed measurements has higher variance than those obtained from traditional measurements. The multiplexed measurements are denoted as $z_{i} \sim \operatorname{Poi}\left(w_{i}^{T} x\right)$ where $w_{i}$ is the $i^{t h}$ row of the multiplexing matrix $W$. We rewrite Poisson observation channel as signal dependent additive noise, i.e., $z_{i} \sim w_{i}^{T} x+\tilde{\kappa}_{i}$, where $\tilde{\kappa}_{i}$ has mean zero and variance $w_{i}^{T} x$, for $i \in\{1,2, \ldots, n\}$. Then, using the Hadamard multiplexing matrix $H$, the multiplexed gain (for ordinary demultiplexing by code matrix inversion) becomes

$$
G(H)=\sqrt{\frac{\operatorname{tr}\left[\mathbb{E}\left(\kappa \kappa^{T}\right)\right]}{\operatorname{tr}\left[\mathbb{E}\left(H^{-1} \tilde{\kappa} \tilde{\kappa}^{T} H^{-T}\right)\right]}}=\sqrt{\frac{n+1}{2 n}} .
$$

Because $n>1$ implies $G<1$, the estimated signal $\hat{x}$ from Hadamard multiplexed data under Poisson noise will always have MSE higher than that obtained from direct measurements. Furthermore, using the proof technique similar to the one used in [9], we conclude that for any type of multiplexing with a non-trivial $(0,1)$ matrix, we have $G<1$ and all forms of multiplexing fail to provide MSE reduction in the case of Poisson noise.

The claims presented in this section only hold, however, when demultiplexing is accomplished using the code matrix inverse. We now generalize the method of demultiplexing using maximum likelihood estimation in the case of non-negatively valued signals. 


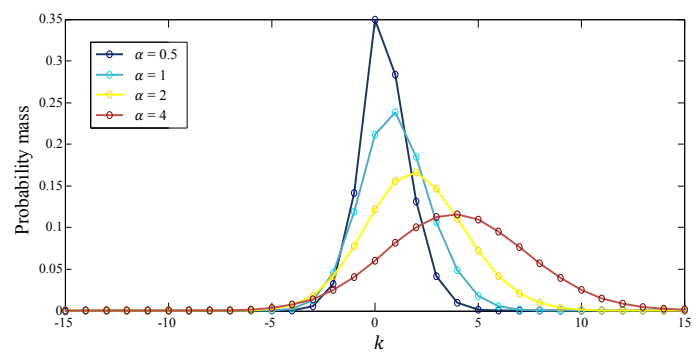

Fig. 3. Probability mass functions of first index variable $p\left(\hat{x}_{1}\right)=$ $S\left(k ; \frac{x_{1}+x_{3}}{2}, \frac{x_{2}}{2}\right)$ for varying values of $x=\alpha \mathbb{1}_{3 \times 1}$.

\section{LOW-RATE POISSON INTENSITY ESTIMATION}

When signal $x$ is unconstrained, the problem of maximum likelihood (ML) estimation of $x$ from multiplexed measurements $z$ under Poisson observation channels is as follows:

$$
\hat{x}=\underset{x}{\arg \max }\left(\prod_{i=1}^{n} \frac{(W x)_{i}^{z_{i}}}{\left(z_{i}\right) !} e^{-(W x)_{i}}\right) .
$$

We now show that, in the unconstrained case, the matrix inversion solution $\hat{x}=W^{-1} z$ is equal the solution of the above optimization problem. To see this, we set $f(x \mid W)$ to be the Poisson likelihood objective function in the above optimization problem. Then, $\hat{x}$ is obtained by minimizing the negative $\log$ of the objective function,

$$
-\log f(x \mid W)=\mathbb{1}^{T}(W x)-\sum_{i=1}^{n} z_{i} \log \left(e_{i}^{T}(W x)\right),
$$

where $\mathbb{1}$ is a vector of ones and $e_{i}$ is a vector with $i$ th entry equal to 1 and other entries equal to 0 . Note that

$$
\nabla_{x}(-\log f(x \mid W))=W^{T} \mathbb{1}-\sum_{i=1}^{n} \frac{\left(W^{T} e_{i}\right) z_{i}}{\left(e_{i}^{T}(W x)\right)}
$$

is equal to zero if $x=W^{-1} z$. Also, $x=W^{-1} z$ is the only optimal solution of $f(W \mid z)$ because of the strong convexity of the likelihood function. This result combined with the theory of multiplexing under Poisson noise presented in section 3 implies that the solution to the unconstrained optimization problem results in higher MSE under Poisson noise compared with traditional imaging.

However, when the signal $x$ is subject to the non-negativity constraint, the matrix inverse solution may not be equal to the solution of the constrained maximum likelihood intensity estimation problem defined as

$$
\hat{x}=\underset{x \geq 0}{\arg \max }\left(\prod_{i=1}^{n} \frac{(W x)_{i}^{z_{i}}}{\left(z_{i}\right) !} e^{-(W x)_{i}}\right) .
$$

We now investigate if multiplexing along with solving the constrained ML intensity estimation problem leads to MSE decrease even in the presence of signal dependent Poisson noise.

When is the non-negativity constraint activated? We illustrate the answer with the simplest non-trivial example. Set $n=3$ and the signal intensity $x$ as a constant vector. Also, set the multiplexer $W$ as the following matrix with full rank:

$$
W=\left[\begin{array}{lll}
1 & 0 & 1 \\
0 & 1 & 1 \\
1 & 1 & 0
\end{array}\right]
$$

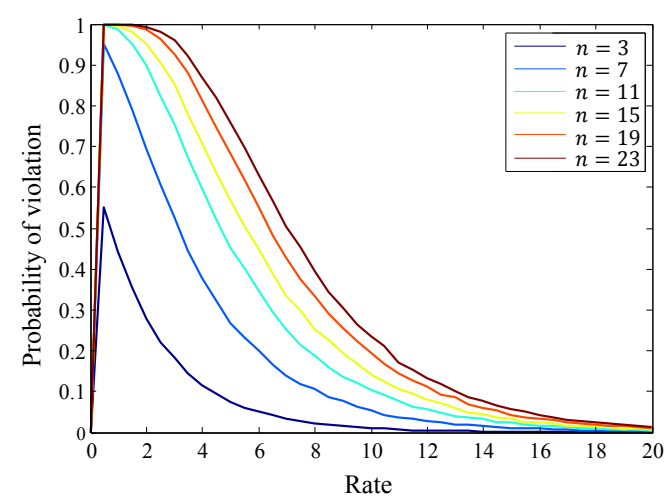

Fig. 4. Probability of any entry of the matrix inverse solution becoming negative with varying rate for several values of $n$.

Since $z \sim \operatorname{Poi}(x)$, the intensity estimate $\hat{x}=W^{-1} z$ is a random vector. We thus have the following expression for the probability density function (pdf) for the first entry of the estimate $\hat{x}$ :

$p\left(\hat{x}_{1}\right)=p\left(\left(W^{-1} z\right)_{1}\right)=p\left(\frac{z_{1}+z_{3}}{2}-\frac{z_{2}}{2}\right)=S\left(k ; \frac{x_{1}+x_{3}}{2}, \frac{x_{2}}{2}\right)$

where $S(k ; \alpha, \beta)=e^{-(\alpha+\beta)}(\alpha / \beta)^{k / 2} I_{|k|}(2 \sqrt{\alpha \beta})$ is the Skellam distribution with $I_{|k|}$ being the modified Bessel function of the first kind [10].

Figure 3 plots $S\left(k ;\left(x_{1}+x_{3}\right) / 2, x_{2} / 2\right)$ vs. $k$ for several constant intensity signals. We see that the area under the plotted curves where $k<0$ decreases as the Poisson noise goes from $x=0.5\left(\mathbb{1}_{3 \times 1}\right)$ to $x=4\left(\mathbb{1}_{3 \times 1}\right)$. In other words, as the Poisson rate decreases, the probability of the first entry of the matrix solution being negative $P\left(\hat{x}_{1}<0\right)$ increases. For $n=3$, because the sum of probabilities of two or more entries going negative is zero, the probability of having a negative entry in the matrix inverse solution is equal to the probability of having an active non-negativity constraint.

In the general case where $n$ is an arbitrary positive integer, what we are then interested in is the probability of at least one of the entries of $\hat{x}=W^{-1} z$ going negative. The probability of activating at least one non-negativity constraint has the following general expression depending on the signal intensity $x$ and the multiplexer $W$ :

$$
\begin{aligned}
& P\left(\left(\hat{x}_{1}<0\right) \cup \ldots \cup\left(\hat{x}_{n}<0\right)\right) \\
& =1-P\left(\left(\hat{x}_{1} \geq 0\right) \cap \ldots \cap\left(\hat{x}_{n} \geq 0\right)\right) \\
& =1-\int_{k_{n} \geq 0} \ldots \int_{k_{1} \geq 0} P\left(\hat{x}_{1}=k_{1}, \ldots, \hat{x}_{n}=k_{n}\right) d k_{1} \ldots d k_{n} \\
& =1-\sum_{W^{-1} c \geq 0} P\left(z_{1}=c_{1}, \ldots, z_{n}=c_{n}\right) \\
& =1-\sum_{W^{-1} c \geq 0} \prod_{i=1}^{n} \frac{(W x)_{i}^{c_{i}}}{c_{i} !} e^{-(W x)_{i}} .
\end{aligned}
$$

Figure 4 plots equation (3) for various values of $n$, and verifies two key claims:

1. if we have high-rate signal $\left(x_{i}>1\right)$, then the ML solution is more likely to be $\hat{x}=W^{-1} z$ and,

2 . in the low-rate regime $\left(x_{i}<1\right)$, the ML solution is most likely not equal to the matrix inverse $\hat{x}=W^{-1} z$.

These results suggest that in the case of low-rate Poisson intensity reconstruction, multiplexing measurements may not always worsen the quality of intensity estimation. 


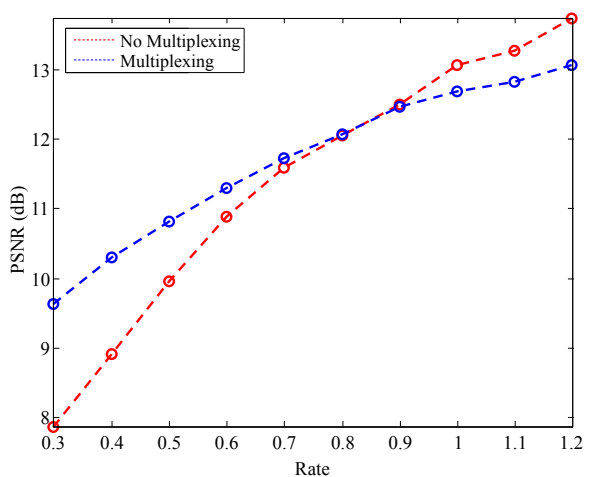

Fig. 5. Plot of PSNR with varying rates.

\section{NUMERICAL EXPERIMENTS}

We examine the MSE reduction when the low-intensity signals under independent Poisson channels are multiplexed. Figure 5 is a peak signal-to-noise ratio (PSNR) comparison plot between reconstructed solutions obtained from non-multiplexed and multiplexed measurements for several constant rates. A Hadamard multiplexing matrix was used to mix a constant positive vector $\left(x_{i}=c, \forall i\right)$ of size $n=63$. The sparse Poisson intensity reconstruction algorithm (SPIRAL) given in [5] was used to reconstruct the maximum likelihood solutions without any regularization. Also, 20 trials of reconstructions are averaged for each simulation. There is a clear improvement when the maximum likelihood solution is obtained after multiplexing under rates below 0.8. At rates above 0.8 , the violation probability decreases and the solution obtained from multiplexed measurements has lower PSNR values than that obtained from measurements without multiplexing.

Figures 6 and 7 show the maximum likelihood intensity estimates (MLIE) from non-multiplexed and multiplexed measurements for both high- and low-rate Poisson channels. These simulations use a multiplexing matrix designed from cyclic codes, which is equal to the adjacency matrix of an undirected 511-cycle graph. Also, knowing that the rate function $x$ is piecewise linear, we generate maximum likelihood intensity estimates with trend filtering (MLIE+TF) to improve the estimation fitting [11].

Plots in Figure 6 show that multiplexing decreases PSNR by $15.3 \mathrm{~dB}$ and confirm the traditional theory that for high-rate intensity reconstruction, the ML solution is the matrix inverse solution and multiplexing is harmful. Also, the trend filtered estimate from non-multiplexed illuminations is higher in PSNR by $11.3 \mathrm{~dB}$ than that from multiplexed illuminations.

On the other hand, Figure 7 plots the same reconstruction results for a low-rate signal and shows that multiplexing improves the reconstruction quality by $1.9 \mathrm{~dB}$. After trend filtering, the estimate solution from multiplexing almost perfectly reconstructs the piecewise constantness of the original intensity function, and gives a PSNR boost of $2 \mathrm{~dB}$ over traditional pointwise acquisition.

\section{CONCLUSIONS}

In this paper, we demonstrate an exception to the view against using multiplexing in the presence of signal dependent Poisson noise. We showed under low-rate Poisson noise, there are clear improvements of SNR boosts when demultiplexing is performed by solving the non-negatively constrained maximum likelihood problem, instead
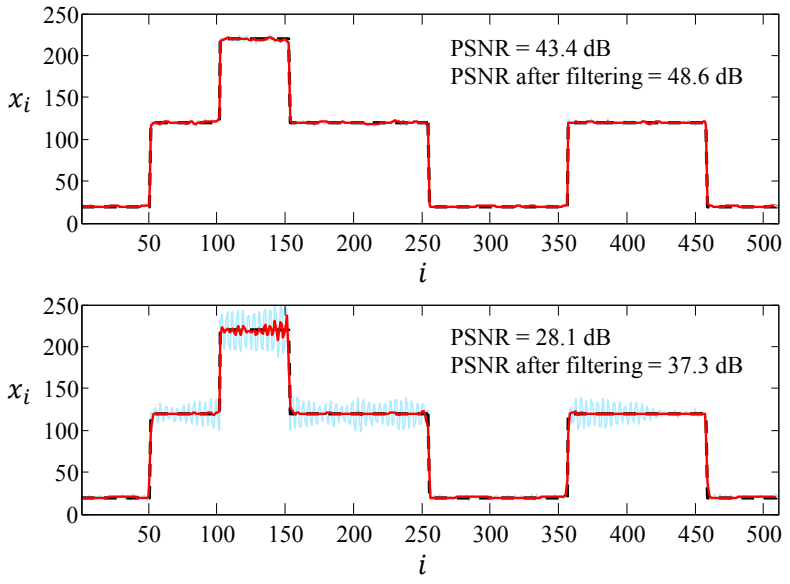

Fig. 6. MLIE (blue line) and MLIE+TF (red line) of a piecewise constant signal $x$ under high-rate Poisson noise from no multiplexing (top) and multiplexing (bottom).
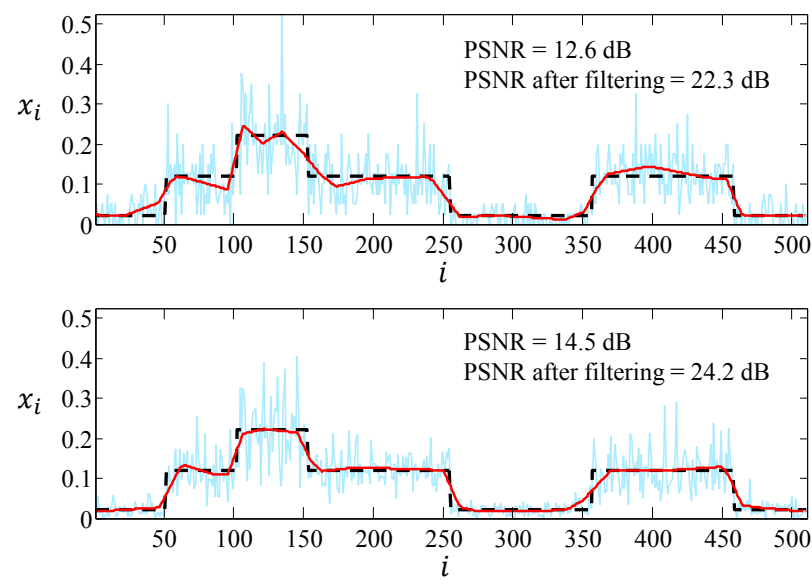

Fig. 7. MLIE (blue line) and MLIE+TF (red line) of a piecewise constant signal $x$ under low-rate Poisson noise from no multiplexing (top) and multiplexing (bottom).

of computing the reconstruction using the matrix inverse solution. We also showed that these improvements are seen when the nonnegativity constraint is active, and using simulations, we observed that there is MSE reduction when the probability of constraint violation is high.

Our results entail theoretical analysis particularly to analyze the performance of different multiplexers under signal dependent Poisson noise. In a more practical setting, our results suggest the use of multiplexing in low light level applications to improve imaging quality.

It is of future interest to analyze the performance of multiplexed imaging when the intensity signal is first degraded through a blurring kernel or a forward imaging operator before being observed through a low-rate Poisson channel. 


\section{REFERENCES}

[1] Y. Y. Schechner, S. K. Nayar, and P. N. Belhumeur, "A theory of multiplexed illumination," in Proc. 9th IEEE Int. Conf. Comput. Vis., 2003, vol. 2, pp. 808-815.

[2] M. Harwit and N. J. Sloane, "Hadamard transform optics," New York: Academic Press, 1979, vol. 1, 1979.

[3] N. Ratner and Y. Y. Schechner, "Illumination multiplexing within fundamental limits," in Proc. IEEE Conf. Comput. Vis. \& Patt. Recog., June 2007.

[4] D. L. Snyder, Random Point Processes, Wiley, 1975.

[5] Z. T. Harmany, R. F. Marcia, and R. M. Willett, "This is SPIRAL-TAP: Sparse Poisson intensity reconstruction algorithms - theory and practice," IEEE Trans. Image Process., vol. 21, no. 3, pp. 1084-1096, Mar. 2012.

[6] S. Rangan, "Generalized approximate message passing for estimation with random linear mixing," in Proc. IEEE Int. Symp. Inform. Theory, Saint Petersburg, Russia, July-Aug. 2011, pp. 2174-2178.

[7] N. J. A. Sloane and M. Harwit, "Masks for Hadamard transform optics, and weighing designs," Appl. Optics, vol. 15, no. 1, pp. 107-114, Jan. 1976.

[8] Y. Y. Schechner, S. K. Nayar, and P. N. Belhumeur, "Multiplexing for optimal lighting," IEEE Trans. Patt. Anal. \& Mach. Intell., vol. 29, no. 8, pp. 1339-1354, Aug. 2007.

[9] N. Ratner, Y. Y. Schechner, and F. Goldberg, "Optimal multiplexed sensing: bounds, conditions and a graph theory link," Optics Exp., vol. 15, no. 25, pp. 17072-17092, Dec. 2007.

[10] A. Alzaid, M. Omair, et al., "On the poisson difference distribution inference and applications," Bulletin of the Malaysian Mathematical Sciences Society, vol. 8, no. 33, pp. 17-45, 2010.

[11] S.-J. Kim, K. Koh, S. Boyd, and D. Gorinevsky, " $\ell_{1}$ trend filtering," SIAM Rev., vol. 51, no. 2, pp. 339-360, Apr. 2009. 